\title{
High dose-rate tandem and ovoid brachytherapy in cervical cancer: dosimetric predictors of adverse events
}

\author{
Kara D. Romano ${ }^{1}$, Colin Hill' ${ }^{1}$ Daniel M. Trifiletti ${ }^{1}$, M. Sean Peach', Bethany J. Horton², Neil Shah', Dylan Campbell', \\ Bruce Libby ${ }^{1}$ and Timothy N. Showalter ${ }^{*^{*}}$ (D)
}

\begin{abstract}
Background: Brachytherapy (BT) is a vital component of the curative treatment of locally advanced cervical cancer. The American Brachytherapy Society has published guidelines for high dose rate (HDR) BT with recommended dose limits. However, recent reports suggest lower doses may be needed to avoid toxicity. The purpose of this study is to investigate incidence and predictive factors influencing gastrointestinal (Gl) and genitourinary (GU) toxicity following HDR intracavitary brachytherapy for locally advanced cervical cancer.

Methods: We retrospectively evaluated a cohort of patients with locally advanced cervical cancer who received CT-based HDR BT. Cumulative doses were calculated using the linear-quadratic model. Statistical analyses were used to investigate clinical and dosimetric predictors of GI and GU toxicity following HDR brachytherapy according to CTCAE v4.0 grading criteria.

Results: Fifty-six women with FIGO IB1 - IVA cervical cancer were included. The overall rate of any GU adverse event (Grade $1+$ ) was 23.3\% ( $n=13)$ and severe adverse events (Grade $3+$ ) was $7.1 \%(n=4)$. Of those, the bladder equivalent dose in 2- Gray (Gy) fractions $\left(E Q D_{2}\right) D_{2 c c}$ was $\geq 80$ for three of the four patients. The overall rate of any $G$ adverse event was $26.8 \%(n=15)$ and the rate of severe adverse events was $14.3 \%(n=8)$. Of those, six of the eight patients had a rectal $E Q D_{2} D_{2 c c} \geq 65$ Gy and seven patients had a sigmoid D2cc $\geq 65 \mathrm{~Gy}$. Amongst clinically meaningful factors for development of adverse events (i.e. diabetes, smoking status, ovoid size, and treatment duration), there were no statistically significant prognostic factors identified.
\end{abstract}

Conclusions: Severe adverse events are observed even with adherence to current ABS guidelines. In the era of recent multi-institutional study results, our data also supports more stringent dosimetric goals. We suggest cumulative D2cc dose limits of: less than 80 Gy for the bladder and less than 65 Gy for the rectum and sigmoid.

\section{Background}

In the United States, cervical cancer continues to represent a sizeable portion of gynecological cancer burden among females, with an estimated 12,820 new cases in 2017 [1]. The definitive treatment for patients with locally advanced cervical cancer involves external beam radiotherapy (EBRT) and concurrent chemotherapy followed by a brachytherapy (BT) boost to achieve optimal treatment outcomes [2]. BT allows for dose escalation of the tumor in a conformal manner that minimizes the toxicity of nearby organs at-risk

\footnotetext{
* Correspondence: tns3b@virginia.edu

${ }^{1}$ Department of Radiation Oncology, University of Virginia School of Medicine, 1240 Lee Street, Box 800383, Charlottesville, VA 22908, USA Full list of author information is available at the end of the article
}

(OARs). This essential role of BT in the curative treatment paradigm has been confirmed by multiple reports, as it confers not only a local control but a survival advantage when compared to cohorts where EBRT is the only radiation treatment modality utilized $[3,4]$.

Over the past three decades, the use of high dose-rate (HDR) BT has substantially increased over low dose-rate (LDR) BT. HDR is now the predominant BT treatment modality in the United States $[3,5]$. Our group recently reported equivalent local control and survival in our experience with LDR and HDR BT [6]. However, the incidence of severe toxicity temporarily increased shortly after the implementation of HDR BT at our institution [6]. The transient rise in severe toxicity represents a

(c) The Author(s). 2018 Open Access This article is distributed under the terms of the Creative Commons Attribution 4.0 International License (http://creativecommons.org/licenses/by/4.0/), which permits unrestricted use, distribution, and 
learning curve in the implementation of new technology and improvements were subsequently made including the incorporation of magnetic resonance imaging (MRI) to the image-guided BT (IGBT) workflow.

The American Brachytherapy Society (ABS) has published guidelines for the delivery of high quality image-guided HDR BT for locally advanced cervical cancer $[7,8]$. Current guidelines recommend 3 dimensional (3D) IGBT with integration of ultra-sound, MRI, or computer assisted tomography to estimate dose to targets and OARs, and to ensure adequate tumor coverage. The total recommended 2 Gray (Gy) equivalent dose $\left(\mathrm{EQD}_{2}\right)$ to target is $80-90$ Gy combined dose from both EBRT and BT. There are variations in acceptable applicators (tandem and ovoid, cylinder, interstitial, etc.), dose specification, and dose fractionation, but dosimetry is required for each fraction due to the large fraction sizes and potential for toxicity. The ABS recommended OAR limits for bladder, rectum, and sigmoid are a $\mathrm{D}_{2 \mathrm{cc}}$ (dose to the hottest dose to $2 \mathrm{~cm}^{3}$ of tissue) of $\leq 90 \mathrm{~Gy}$, $\leq$ $75 \mathrm{~Gy}$, and $\leq 75$ Gy, respectively $[7,8]$.

Similarly, the Group Europeen de Curietherapie (GEC) - European Society for Radiotherapy and Oncology (ESTRO) (GEC-ESTRO) gynecological (GYN) Working Group has published recommendations for the treatment of cervical cancer with IGBT [9]. Based on recent publications from the prospective multi-center, "European study on MRI-guided brachytherapy in locally advanced cervical cancer" (EMBRACE), study and retrospective "RetroEMBRACE," the currently accruing EMBRACE II protocol OAR planning aims are $\mathrm{D}_{2 \mathrm{cc}}<80$ Gy for bladder, < 65 Gy for rectum, $<65$ Gy for recto-vaginal point, and $<70$ Gy for sigmoid and bowel [10, 11].

The purpose of this retrospective series is to report on our institution's experience with image-guided HDR brachytherapy in the framework of the current guidelines set forth by the ABS and in light of the recent EMBRACE collaborative results supporting more stringent dose recommendations [7].

\section{Methods}

\section{Patient population}

After obtaining approval from the institutional review board, retrospective data was collected for patients with cervical cancer who received HDR tandem and ovoid BT and CT-based treatment planning from 2012 to 2014 to assess for dosimetric predictors of toxicity Clinical stages IB - IVA via the International Federation of Gynecology and Obstetrics (FIGO) staging criteria were included if dosimetry and follow up toxicity information was available during the specified time period. Patients were excluded if they received LDR BT or interstitial BT.

Baseline clinical prognostic factors were recorded including: age, stage (FIGO and tumor, nodal, metastasis
(TMN) staging), Eastern Cooperative Oncology Group (ECOG) performance status, histology, clinical tumor size at diagnosis, diabetic history, and smoking status. Treatment-related variables including ovoid size and treatment duration were also chronicled. Clinical adverse events, if present, was scored by chart review as Grade $1-4$ as per the common terminology criteria for adverse events (CTCAE) v4.03 for both acute and chronic conditions beginning from the start of radiation therapy [12]. Severe adverse events were defined as Grade 3 or higher.

\section{Treatment}

\section{External beam radiation therapy and chemotherapy}

In 2012, our institution transitioned from LDR to a 3-D IGBT HDR program, and we have previously described our technique in detail [13]. In brief, the treatment of cervical cancer at our institution involves EBRT to a total dose of 45-50.4 Gray (Gy) in 1.8-2 Gy/fraction with concurrent cisplatin-based chemotherapy, if indicated, followed by intra-cavitary tandem \& ovoid BT. BT is delivered after the EBRT course is complete, or in the last week of pelvic EBRT. The EBRT volume includes at least the whole pelvis with extended field technique included for more advanced disease such as positive pelvic or para-aortic nodes. Intensity modulated (IMRT) and 3-D techniques are used with 6-15 MV photon therapy. Positive nodes and/or involved parametria receive an additional boost dose of EBRT. We do not administer HDR BT on the same day as chemotherapy, and the goal is to complete all treatment within 56 days.

\section{HDR brachytherapy}

HDR BT is delivered with an ${ }^{192} \mathrm{Ir}$ source and a single tandem and two symmetric ovoid applicators. The applicators are placed in a dedicated brachytherapy procedure suite with an in-room CT-on-rails system and HDR afterloader. A Smit sleeve is placed under anesthesia at the time of the first fraction of BT and remains in place through the final fraction. After placement of the applicators, all patients undergo computed tomography (CT) simulation for treatment planning. Beginning in 2014, our institution implemented a magnetic resonance imaging (MRI) integrated workflow for asynchronous MRI-based IGBT. Please see prior institutional publication for workflow details [13]. Pelvic MRI is obtained between the first and second fractions of BT in parallel and orthogonal planes of the Smit sleeve applicator. The MRI T2 weighted sequences define the extent of residual tumor and is co-registered along the plane of the Smit sleeve with the treatment planning CT for each subsequent fraction of $\mathrm{BT}$. In accordance with $\mathrm{ABS}$ guidelines, the total HDR treatment dose is $25-30$ Gy in 4-5 separate fractions (most commonly 5 fractions of 5-5.5 Gy each) with no more than two fractions per week and 
never on consecutive days. The goal for total tumor $\mathrm{EQD}_{2}$ is $80-90 \mathrm{~Gy}$.

Manual dose optimization is performed on a standardized plan for individualized treatment planning. We start with a customary loading pattern for traditional Point A prescription, and then modify dwell positions and times to ensure coverage of the high-risk clinical target volume (HR-CTV) and the MRI-defined residual gross tumor volume (GTV-BT) and to minimize doses to the organs-at-risk (OAR). Since MRI is not available until after the first fraction of BT, an estimated HR-CTV is contoured on the planning CT even though an MRI is not available. Although HR-CTV is a concept based on MRI-based target delineation, contouring on CT for the first fraction permits us to track the cumulative doses in our clinical practice. To allow for treatment optimization based on daily OAR position and distension, critical OARs are contoured as a whole organ on the $\mathrm{CT}$ data set prior to treatment delivery for each fraction. A cumulative dose summation worksheet is used to track cumulative biologically effective doses (BED) and $\mathrm{EQD}_{2}$ from EBRT and each fraction of HDR BT for the targets and OARs. The alpha-beta ratio is assumed to be 10 for the tumor and 3 for normal tissues. Target doses include HR-CTV and GTV-BT $\mathrm{V}_{100}, \mathrm{D}_{90}$, and $\mathrm{D}_{100}$. OAR doses recorded include: bladder, rectum, sigmoid, and bowel $\mathrm{D}_{0.1 \mathrm{cc}}, \mathrm{D}_{1 \mathrm{cc}}$, and $\mathrm{D}_{2 \mathrm{cc}}$.

\section{Statistical analysis}

Clinical, treatment-related, and toxicity factors were reported as categorical and continuous variables, as appropriate. Fisher's exact test and the Wilcoxon MannWhitney test were used to assess differences in clinical and treatment characteristics between patient groups defined by those with adverse event grades of 2 or less and 3 or greater, with $p$-values presented in Table 1. Proportional hazard models and log-rank tests were used to identify clinical and dosimetric predictors, given in Table 1, for toxicity following HDR BT according to CTCAE criteria. All statistical analyses were performed using commercially available statistical software (SAS, version 9.4; SAS Institute Inc., Cary, NC).

\section{Results}

\section{Clinical and dosimetric characteristics}

Fifty-six (56) women with FIGO stage IB1 - IVA cervical cancer treated with HDR BT at our institution met inclusion criteria. Clinical and treatment characteristics are summarized in Table 1 . The majority of patients in this cohort had FIGO stage IIB or IIIB (55.4\%) squamous cell carcinoma and 39 patients $(70.0 \%)$ had positive pelvic nodes noted on staging studies. Overall performance status was good; however, 3 patients were included with ECOG score of 3 or higher. The median follow up time, amongst patients alive at time of analysis, is 7 months (0-26 months).

The clinical and treatment characteristics of those without a severe acute adverse event $(\leq$ Grade 2 ) or with a severe adverse event ( $\geq$ Grade 3 ) are presented in Table 1. Note that the estimated clinical tumor size at diagnosis for each group is similar $(5.5 \mathrm{vs} 5.4 \mathrm{~cm}$ ). Patients with severe toxicity were more likely to be current smokers $(71 \%$ vs $35 \%)$, have longer treatment duration (57\% over 56 days versus $47 \%$ ), more advanced T stage (all T2b or greater), and were slightly younger in age (52 vs. 54 years).

\section{Bladder toxicity}

Overall, thirteen patients (23.3\%) developed a genitourinary (GU) adverse event of any grade. Four patients (7.1\%) experienced a severe (defined as Grade 3 or greater) GU adverse event. Among patients with Grade 3+ GU toxicity, 1 (25\%) had bladder D2cc $\leq 80$ Gy, 1 (25\%) had bladder D2cc 80-90 Gy, and $2(50 \%)>90$ Gy. A cumulative bladder $D_{2 \mathrm{cc}}$ threshold of 90 Gy would permit a $5.6 \%$ rate of grade $3+\mathrm{GU}$ toxicity, compared to a $3.6 \%$ rate of grade $3+\mathrm{GU}$ toxicity if the threshold is lowered to 80 Gy. In Table 2, GU and gastrointestinal (GI) adverse events are reported by grade according to the EQD $D_{2} \mathrm{D}_{2 \mathrm{cc}}$ for bladder, rectum, and sigmoid. Fig. 1 depicts the grade of adverse event by recommended dose constraints for bladder (A), rectum (B) and sigmoid (C).

\section{Rectal/sigmoid toxicity}

Overall, fifteen patients (26.8\%) developed a GI adverse event of any grade. Eight patients (14.3\%) developed a severe GI adverse event. Of the 8 patients with Grade 3+ GI toxicity, 2 had rectal $D_{2 c c}$ of 65 Gy or less $(25 \%), 3$ had doses between 65 and 75 Gy (37.5\%), and 3 had doses greater than 75 Gy (37.5\%). A cumulative rectal $\mathrm{D}_{2 \mathrm{cc}}$ threshold of 75 Gy would result in a $16.1 \%$ rate of Grade 3+ GI toxicity. A cumulative sigmoid $D_{2 c c}$ threshold of 75 Gy would permit a $14.6 \%$ rate of grade 3+ GI toxicity compared to a permitted rate of $4.5 \%$ if the threshold is lowered to $65 \mathrm{~Gy}$.

\section{Prognostic factors for toxicity}

Logistic regression models were used to model GU and GI adverse events. The models were adjusted by dose to related organs (bladder for GU toxicity and rectum/sigmoid for GI toxicity) in additional 5 Gy increments. No covariates were statistically significant $(p=0.276$ for bladder, $p=0.361$ for rectum, and $p=0.092$ for sigmoid). Several other factors of interest were assessed for their prognostic effects on time to developing Grade 3+ adverse events, including diabetes, smoking status, ovoid size, and treatment duration. Log-rank testing did not 
Table 1 Baseline characteristics of all patients and by adverse event grade

\begin{tabular}{|c|c|c|c|c|c|c|}
\hline & \multirow{2}{*}{$\begin{array}{l}\text { All } \\
n\end{array}$} & \multicolumn{2}{|c|}{$\begin{array}{l}2 \text { or less } \\
n=49\end{array}$} & \multicolumn{2}{|c|}{$\begin{array}{l}3 \text { or greater } \\
n=7\end{array}$} & \multirow[b]{2}{*}{$p$-value } \\
\hline & & $\bar{n}$ & $\begin{array}{l}\% \text { or } \\
95 \% \mathrm{Cl}\end{array}$ & $\bar{n}$ & $\%$ or $95 \% \mathrm{Cl}$ & \\
\hline \multicolumn{7}{|l|}{ Clinical characteristics } \\
\hline Age at start of radiation treatment ${ }^{1}$ & 53.4 & 53.6 & $49.6-57.8$ & 51.8 & $40.7-62.9$ & 0.961 \\
\hline Histology & & 44 & 89.8 & 7 & 100 & 1.00 \\
\hline Squamous cell carcinoma & 51 & & & & & \\
\hline Adenocarcinoma & 4 & 4 & 8.2 & 0 & 0 & \\
\hline Adenosquamous & 1 & 1 & 2.0 & 0 & 0 & \\
\hline FIGO stage & & 9 & 18.4 & 0 & 0 & 0.236 \\
\hline |B1 & 9 & & & & & \\
\hline IB2 & 14 & 13 & 26.5 & 1 & 14.3 & \\
\hline$\| \mathrm{A} 1$ & 0 & 0 & 0 & 0 & 0 & \\
\hline$\| \mathrm{A} 2$ & 1 & 1 & 2.0 & 0 & 0 & \\
\hline$\| B$ & 16 & 13 & 26.5 & 3 & 42.9 & \\
\hline$\| \mathrm{A}$ & 0 & 0 & 0 & 0 & 0 & \\
\hline$\| \mathrm{II}$ & 15 & 13 & 26.5 & 2 & 28.6 & \\
\hline IVA & 1 & 0 & 0 & 1 & 14.3 & \\
\hline N stage & & 15 & 30.6 & 2 & 28.6 & 1.00 \\
\hline No & 17 & & & & & \\
\hline N1 - pelvic nodes & 39 & 34 & 69.4 & 5 & 71.4 & \\
\hline M stage & & 39 & 79.6 & 7 & 100 & 0.719 \\
\hline MO & 46 & & & & & \\
\hline M1 - para-aortic nodes & 8 & 8 & 16.3 & 0 & & \\
\hline Unknown & 2 & 2 & 4.1 & 0 & & \\
\hline Clinical tumor size at diagnosis $(\mathrm{cm})^{1}$ & 5.4 & 5.4 & $4.6-6.1$ & 5.5 & $4.3-6.7$ & 0.742 \\
\hline ECOG & & 31 & 63.3 & 7 & 100 & 0.558 \\
\hline 0 & 38 & & & & & \\
\hline 1 & 9 & 9 & 18.4 & 0 & 0 & \\
\hline 2 & 6 & 6 & 12.2 & 0 & 0 & \\
\hline 3 & 2 & 2 & 4.1 & 0 & 0 & \\
\hline 4 & 1 & 1 & 2.0 & 0 & 0 & \\
\hline \multicolumn{7}{|l|}{ Treatment characteristics } \\
\hline Acute toxicity grade & & & & & & - \\
\hline 0 & 45 & 45 & 91.8 & 0 & 0 & \\
\hline 1 & 3 & 3 & 6.1 & 0 & 0 & \\
\hline 2 & 1 & 1 & 2.0 & 0 & 0 & \\
\hline 3 & 5 & 0 & 0 & 5 & 71.4 & \\
\hline 4 & 2 & 0 & 0 & 2 & 28.6 & \\
\hline Chronic toxicity grade & & & & & & 0.008 \\
\hline 0 & 36 & 35 & 71.4 & 1 & 14.3 & \\
\hline 1 & 5 & 3 & 6.1 & 2 & 28.6 & \\
\hline 2 & 5 & 4 & 8.2 & 1 & 14.3 & \\
\hline 3 & 10 & 7 & 14.3 & 3 & 42.9 & \\
\hline 4 & 0 & 0 & 0 & 0 & 0 & \\
\hline
\end{tabular}


Table 1 Baseline characteristics of all patients and by adverse event grade (Continued)

\begin{tabular}{|c|c|c|c|c|c|c|}
\hline & All & & & & & \\
\hline & $\mathrm{n}$ & $\bar{n}$ & $\begin{array}{l}\% \text { or } \\
95 \% \mathrm{Cl}\end{array}$ & $\bar{n}$ & $\%$ or $95 \% \mathrm{Cl}$ & $p$-value \\
\hline Treatment Response & & & & & & 0.914 \\
\hline Stable Disease & 5 & 5 & 10.2 & 0 & 0 & \\
\hline Complete Response & 20 & 18 & 36.7 & 2 & 28.6 & \\
\hline Partial Response & 21 & 19 & 38.8 & 3 & 42.9 & \\
\hline Progression & 5 & 4 & 8.2 & 1 & 14.3 & \\
\hline Unknown & 4 & 3 & 6.1 & 1 & 14.3 & \\
\hline Treatment Duration & & & & & & 0.700 \\
\hline Greater than 56 days & 27 & 23 & 46.9 & 4 & 57.1 & \\
\hline 56 days or less & 29 & 26 & 53.1 & 3 & 42.9 & \\
\hline Ovoid Size & & & & & & 0.236 \\
\hline Large & 1 & 1 & 2.0 & 0 & 0 & \\
\hline Medium & 15 & 15 & 30.6 & 0 & 0 & \\
\hline Mini & 8 & 6 & 12.2 & 2 & 28.6 & \\
\hline Small & 30 & 25 & 51.0 & 5 & 71.4 & \\
\hline Unknown & 2 & 2 & 4.1 & 0 & 0 & \\
\hline Comorbidities & & & & & & \\
\hline Smoker & & 24 & 49.0 & 1 & 14.3 & 0.149 \\
\hline Never & 25 & & & & & \\
\hline Former & 9 & 8 & 16.3 & 1 & 14.3 & \\
\hline Current & 22 & 17 & 34.7 & 5 & 71.4 & \\
\hline Diabetes Mellitus & & & & & & 1.00 \\
\hline No & 50 & 43 & 87.8 & 7 & 100 & \\
\hline Yes & 6 & 6 & 12.2 & 0 & 0 & \\
\hline
\end{tabular}

ECOG Eastern Cooperative Oncology Group Performance Status, FIGO International Federation of Gynecology and Obstetrics

${ }^{1}$ Mean

show any of the curves to approach statistical significance ( $p$-values $>0.05$ ).

\section{Dosimetry}

Figure 2 depicts in a histogram the percentage of patients receiving specified EQD2 doses.

\section{Discussion}

The ABS guidelines in 2012 recommended EQD2 $\mathrm{D}_{2 \mathrm{cc}}$ dosimetric thresholds of 90 Gy for the bladder and 75 Gy for the rectum and the sigmoid to minimize toxicity [7]. Our data demonstrates that high rates of toxicity are still possible in clinical practice even with adherence to the current ABS guidelines for dose limits to normal tissues. Based on our observed toxicities, we suggest more stringent cumulative D2cc dosimetric goals than in current ABS guidelines: specifically, less than $80 \mathrm{~Gy}$ for the bladder and less than $65 \mathrm{~Gy}$ (or as low as achievable without compromising local control) for the rectum and sigmoid.
Our study is relevant in light of other recent multi-institutional publications also supporting more stringent dose limits. The prospective multi-center EMBRACE study was initiated in 2008 utilizing MRI-guided BT prior to an understanding of specific OAR dose constraints in this 3-D era. Subsequent reports from EMBRACE and the retrospective RetroEMRACE studies report an overall severe toxicity (Grade 3 of higher) of around $3-7 \%$ per organ $[10,11]$. A dose-volume effect for rectum was established with $D_{2 \mathrm{cc}} \leq 65$ Gy correlating with more minor and less frequent rectal morbidity where rectal $D_{2 c c} \geq 75$ Gy was associated with more severe rectal morbidity and risk of fistula [14]. Significant dose effect curves for bladder morbidity have also been reported with a $5 \%$ risk of late urinary morbidity for $\mathrm{D}_{2 \mathrm{cc}} 60-70$ Gy [15]. Based on these findings, the currently accruing EMBRACE II protocol OAR planning aims are $\mathrm{D}_{2 \mathrm{cc}}<80 \mathrm{~Gy}$ for bladder, $<65$ Gy for rectum, $<$ 65 Gy for recto-vaginal point, and $<70$ Gy for sigmoid and bowel [10]. 
Table 2 Frequency of adverse event by recommended dose category

\begin{tabular}{|c|c|c|c|c|c|c|c|c|}
\hline & \multicolumn{6}{|c|}{ Bladder total EQD2 $\mathrm{D}_{2 \mathrm{cc}}$} & \multirow{2}{*}{\multicolumn{2}{|c|}{ All }} \\
\hline & \multicolumn{2}{|c|}{0 to 80} & \multicolumn{2}{|c|}{80 to 90} & \multicolumn{2}{|c|}{$>90$} & & \\
\hline & $n$ & $\%$ & $n$ & $\%$ & $n$ & $\%$ & $n$ & $\%$ \\
\hline GU Toxicity Grade & 24 & 85.7 & 5 & 62.5 & 14 & 70.0 & 43 & 76.8 \\
\hline \multicolumn{9}{|l|}{0} \\
\hline 1 & 2 & 7.1 & 1 & 12.5 & 1 & 5.0 & 4 & 7.1 \\
\hline 2 & 1 & 3.6 & 1 & 12.5 & 3 & 15.0 & 5 & 8.9 \\
\hline 3 & 0 & 0.0 & 1 & 12.5 & 2 & 10.0 & 3 & 5.4 \\
\hline 4 & 1 & 3.6 & 0 & 0.0 & 0 & 0.0 & 1 & 1.8 \\
\hline \multirow[t]{4}{*}{ All } & 28 & 100.0 & 8 & 100.0 & 20 & 100.0 & 56 & 100.0 \\
\hline & \multicolumn{6}{|c|}{ Rectal total EQD2 $D_{2 c c}$} & \multicolumn{2}{|l|}{ All } \\
\hline & \multicolumn{2}{|c|}{0 to 65} & \multicolumn{2}{|c|}{65 to 75} & \multicolumn{2}{|c|}{$>75$} & & \\
\hline & $\mathrm{n}$ & $\%$ & $\mathrm{n}$ & $\%$ & $\mathrm{n}$ & $\%$ & $n$ & $\%$ \\
\hline Gl Toxicity Grade & 8 & 72.7 & 17 & 85.0 & 16 & 64.0 & 41 & 73.2 \\
\hline \multicolumn{9}{|l|}{0} \\
\hline 1 & 0 & 0.0 & 0 & 0.0 & 4 & 16.0 & 4 & 7.1 \\
\hline 2 & 1 & 9.1 & 0 & 0.0 & 2 & 8.0 & 3 & 5.4 \\
\hline 3 & 1 & 9.1 & 3 & 15.0 & 3 & 12.0 & 7 & 12.5 \\
\hline 4 & 1 & 9.1 & 0 & 0.0 & 0 & 0.0 & 1 & 1.8 \\
\hline \multirow[t]{4}{*}{ All } & 11 & 100.0 & 20 & 100.0 & 25 & 100.0 & 56 & 100.0 \\
\hline & \multicolumn{6}{|c|}{ Sigmoid total EQD2 $D_{2 c c}$} & \multicolumn{2}{|l|}{ All } \\
\hline & \multicolumn{2}{|c|}{0 to 65} & \multicolumn{2}{|c|}{65 to 75} & \multicolumn{2}{|c|}{$>75$} & & \\
\hline & $n$ & $\%$ & $n$ & $\%$ & $n$ & $\%$ & $n$ & $\%$ \\
\hline Gl Toxicity Grade & 20 & 90.9 & 11 & 57.9 & 10 & 66.7 & 41 & 73.2 \\
\hline \multicolumn{9}{|l|}{0} \\
\hline 1 & 1 & 4.5 & 1 & 5.3 & 2 & 13.3 & 4 & 7.1 \\
\hline 2 & 0 & 0.0 & 2 & 10.5 & 1 & 6.7 & 3 & 5.4 \\
\hline 3 & 1 & 4.5 & 5 & 26.3 & 1 & 6.7 & 7 & 12.5 \\
\hline 4 & 0 & 0.0 & 0 & 0.0 & 1 & 6.7 & 1 & 1.8 \\
\hline All & 22 & 100.0 & 19 & 100.0 & 15 & 100.0 & 56 & 100.0 \\
\hline
\end{tabular}

GU Genitorinary, GI Gastrointestinal, EQD2 Equivalent dose in 2 Gy fractions assuming $\alpha / \beta=3 \mathrm{~Gy}, D_{2 c c}$ the most irradiated $2 \mathrm{~cm}^{3}$ of normal tissue volume

There are inherent challenges to comparing toxicity outcomes across different series. The American Brachytherapy Task Group conducted a meta-analysis of retrospective and prospective series from 2000 to 2015 [16]. In their report, the late GI and GU toxicities ranged from 4 to $11 \%$ and $1-6 \%$, respectively, for radiation alone and $1-$ $11 \%$ and $2-20 \%$, respectively, for chemo-radiation. The authors note that only a limited number of image-based brachytherapy (IGBT) series reported toxicity. Further, there is a the lack of consistency in toxicity scoring systems and other confounding variables such as differences in treatment planning, which may lead to significant variation in dosimetric outcomes to the surrounding OAR. Our rate of severe GI adverse events is higher than those summarized in this meta-analysis; which may be related to the above-mentioned limitations with differences in scoring, reporting, and the inclusion of patients in the pre-MRI based imaging era.

\section{Rectal/sigmoid toxicity}

We found a high rate of severe GI toxicity for rectal and sigmoid $\mathrm{D}_{2 \mathrm{cc}}$ over $65-75 \mathrm{~Gy}$. Other series in the era of IGBT treatment planning have validated volume-based dose parameters EQD2 $\mathrm{D}_{2 \mathrm{cc}}$ as a reliable parameter for predicting rectal and bladder toxicities [15]. Increased rates of rectal toxicity (acute, late, and/or both) have been demonstrated with a $\mathrm{D}_{2 \mathrm{cc}}$ of $78 \mathrm{~Gy}$ and of $65 \mathrm{~Gy}$ $[15,17,18]$. Another retrospective series of patients identified a significant relationship between late rectal toxicity and a cumulative rectal dose in EQD2 greater than 65 Gy. Taking into account these various reports in the literature and our rectal toxicity rate, it is reasonable to suggest that a more stringent cumulative $D_{2 c c}$ threshold would lead to more optimal toxicity outcomes.

The dose constraints for the sigmoid are still unclear. There was no sigmoid 2-D correlate and clinical data for sigmoid are not as widely reported. While there has not been a sigmoid dose-volume relationship established in the previously published literature, this cohort demonstrates an association between sigmoid doses $>65$ Gy and high rates of severe toxicity. Thus, current protocols including EMBRACE II indicate respecting the same OAR dose constraint for rectum and sigmoid [19].

\section{Bladder toxicity}

A well-defined dose-effect relationship for bladder doses and urinary morbidity in the treatment of cervical cancer has been demonstrated. $\mathrm{D}_{2 \mathrm{cc}}$ thresholds of $100 \mathrm{~Gy}$ and 95 Gy have been shown to correlate with bladder toxicity in previously reported series $[15,20]$. However, another series of IGBT reporting a $5.9 \%$ rate of late grade $3+\mathrm{GU}$ morbidity but could not identity a relationship between $\mathrm{GU}$ toxicity and the bladder $\mathrm{D}_{2 \mathrm{cc}}[17]$. An additional point to consider with bladder OAR is the highly distensible nature of the organ and that dose-volume histogram (DVH)-summed parameters may not always accurately estimate bladder dose [21]. Review of the literature clearly suggests that the limit of the bladder dose threshold has yet to be clearly defined, but our results suggest considering the fact that a $D_{2 c c}$ dose of 80 Gy will permit more acceptable toxicity rates than the current dosimetric guidelines for the bladder. The EMBRACE II protocol defines the bladder contour as the outer wall including the bladder neck with a planning aim of $\mathrm{D}_{2 \mathrm{cc}}<80$ Gy with a hard limit of < 90 Gy [19].

\section{Vaginal toxicity}

Until recently, late gynecologic toxicity was rarely reported. In a recent meta-analysis, only 1 of 16 prospective 

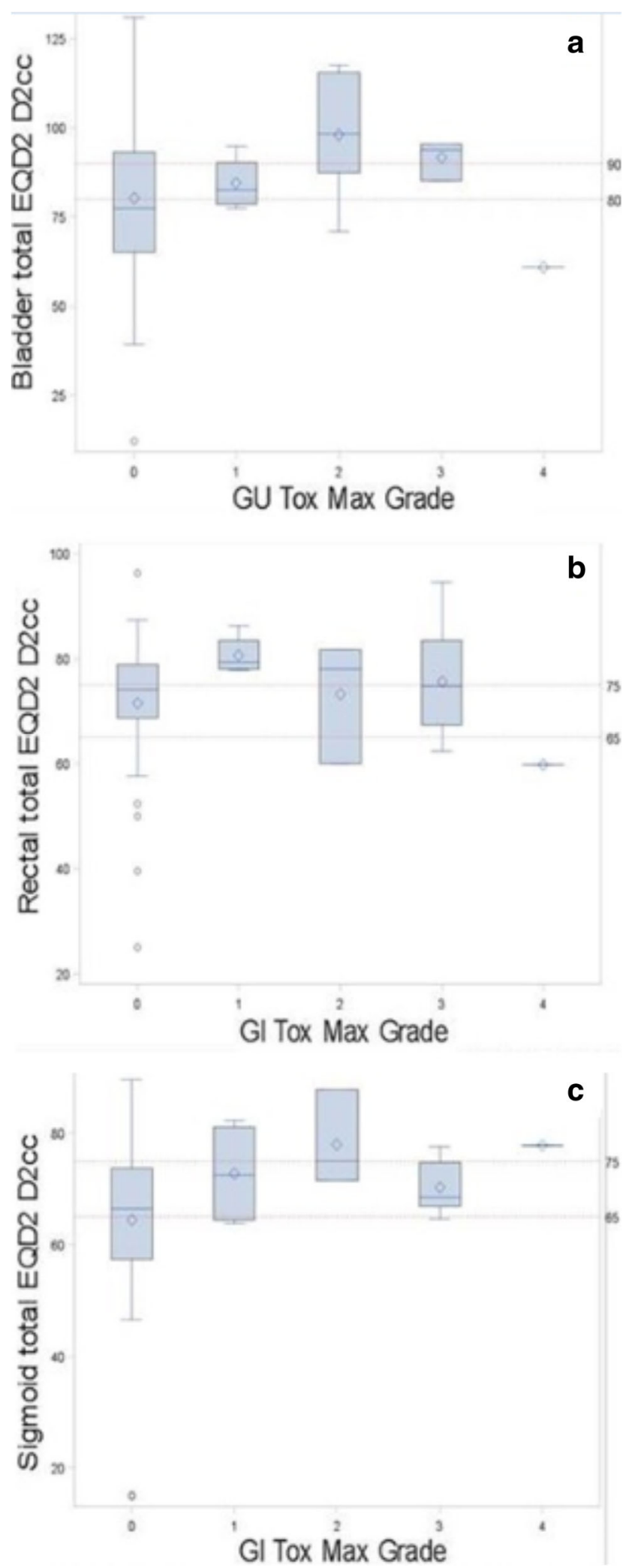

Fig. 1 Box plots with adverse event and recommended dose constraints for bladder a, rectum (b) and sigmoid (c) 

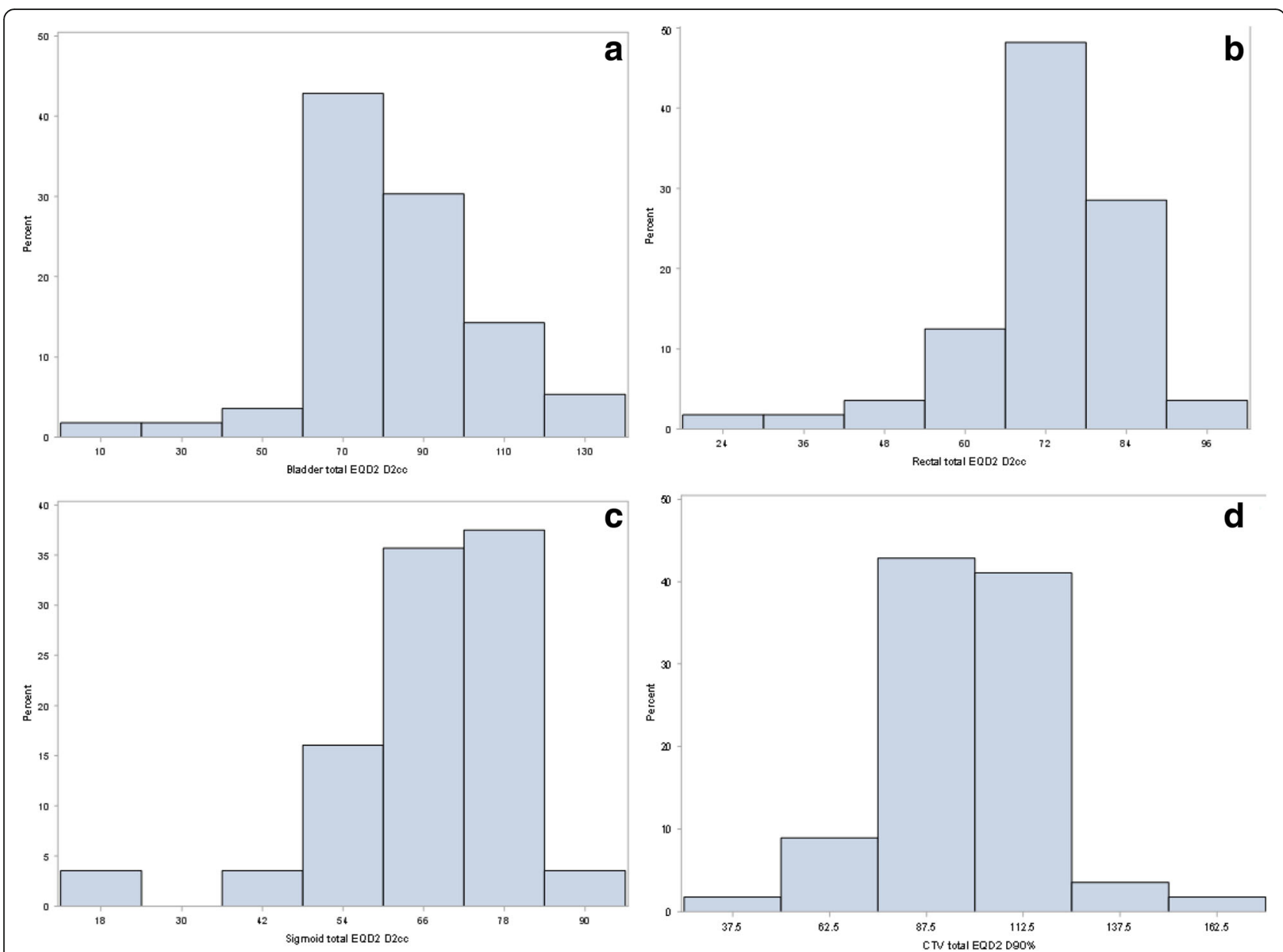

Fig. 2 Histograms percent of patients with equivalent dose in 2 Gy fractions (EQD2Gy) for bladder a, rectum b, sigmoid $\mathbf{c}$, and CTV (d)

trials reported late gynecologic toxicity with a mean rate of $16 \%$ following RT alone. From EMRACE we learned that recto-vaginal point dose correlated with vaginal stenosis with 65 Gy leading to 20\% vaginal stenosis [22]. While vaginal toxicity is not reported here, these data again support tighter dose constraints to limit toxicity.

\section{Image guidance}

The incorporation of IGBT for applicator placement and treatment planning has generated the opportunity for much advancements in target coverage and normal tissue sparing. Ideal dosimetric distributions with HDR can be achieved only if an optimal optimization process accompanies proper applicator placement. With the evolution of treatment planning processes from prescribing a dose to a fixed point to 3-D treatment planning, the development of individualized dosimetric distributions that adequately cover the HR-CTV while sparing the OAR can be challenging. However, multiple studies have demonstrated the benefit of 3-D treatment planning with improvements in local control and survival endpoints and better morbidity outcomes [16, 23, 24]. Due to its demonstrated benefits, a growing number of institutions have made the switch to IGBT in recent years, but it has yet to be universally implemented due to the complexity and time-consuming nature of IGBT [16]. For institutions that have the capability to perform hybrid CT-MRI or MRI-based IGBT, the superior target-volume delineation that is attainable with MRI [9, 25] has the potential to allow for compliance to more stringent OAR dose thresholds, potentially allowing for better morbidity.

\section{Limitations}

The limitations of this study are inherent to the retrospective nature of a single institution's experience. Moreover, we did not control for variations in applicator placement accuracy, target volume size, or differences in systemic therapy. We attempted to identify clinical factors prognostic for whether patients would develop adverse events but were unable to do so due to the relatively small sample size of patients in our cohort experiencing grade $>3$ adverse events. As mentioned, scoring systems for toxicity are inconsistent across series 
and other series may report their data using different toxicity scales.

We were able to identify a clinically meaningful relationship between dosimetric limits for normal tissues and toxicity, but perhaps due to small sample sizes we were unable to also identify clinical factors that would be significantly prognostic of whether patients would experience severe adverse events. For example, additional factors that may influence toxicity in this patient cohort that were not reported here include obesity, co-morbidity index (heart disease, COPD, hypertension, etc.), and baseline GU/GU functional repots. Future studies can incorporate these potentially important factors.

\section{Future directions}

The currently accruing EMBRACE II study is evaluating image guided adaptive BT in a prospective multi-institutional setting [19]. The results of this trial will help us to further understand the dose and volume effect relationships of OARs and related morbidity. One of the ways this trial will improve upon prior experiences is through the implementation of a wider variety of applicator types (intracavitary and interstitial), which will allow for greater modulating ability of source position and dwell times. Additionally, organ manipulation (i.e bladder filling or rectal retraction) can confer a gainful benefit to achieving better dosimetric distributions [26, 27]. Continued development of applicators to displace normal tissues has the potential to further improve normal tissue doses and related morbidity. Despite the importance of toxicity and the need to prioritize optimization of dosimetry to spare organs-at-risk, it is also important to remember that delivering sufficient dose to the cervical tumor must remain the top priority in the curative treatment of cervical cancer. In some situations, therefore, threshold doses to adjacent organs may be exceeded in order to achieve adequate tumore dose.

\section{Conclusion}

In clinical practice, a relatively high rate of GI and GU toxicity is still possible even with adherence to current ABS guidelines for dosimetric objectives. We suggest considering more stringent cumulative D2cc dosimetric goals than in current ABS guidelines: less than 80 Gy for the bladder and less than 65 Gy (or as low as achievable without compromising local control) for the rectum and sigmoid.

\footnotetext{
Abbreviations

3D: 3-dimensional; ABS: American Brachytherapy Society; BED: Biologically effective dose; BT: Brachytherapy; CTCAE: Common terminology criteria for adverse events; $D_{0.1 c c}, D_{1 c c}, D_{2 c c}$ : Dose delivered to the hottest $0.1 \mathrm{cc}, 1 \mathrm{cc}$, and $2 \mathrm{cc}$ of an organ at-risk; $\mathrm{D}_{90}, \mathrm{D}_{100}$ : Dose delivered to the hottest 90 and $100 \%$ of the target volume; EBRT: External beam radiation therapy;

ECOG: Eastern Cooperative Oncology Group; EMBRACE: European study on
}

MRI-guided brachytherapy in locally advanced cervical cancer; EQD $2: 2$ Gray equivalent dose; FIGO: International Federation of Gynecology and

Obstetrics; GEC-ESTRO: Group Europeen de Curietherapie (GEC) - European Society for Radiotherapy and Oncology (ESTRO); GTV-BT: MRI-defined gross tumor volume at time of brachytherapy; Gy: Gray; GYN: Gynecological; HDR: High dose-rate; HR-CTV: High-risk clinical target volume; IGBT: Imageguided brachytherapy; IMRT: Intensity-modulated radiation therapy; LDR: Low dose-rate; MRI: Magnetic resonance imaging; OAR: Organ at-risk;

$V_{100}$ : Volume of target volume receiving $100 \%$ of the prescription dose

Availability of data and materials

Please contact author for data requests.

Authors' contributions

$\mathrm{KDR}, \mathrm{CH}, \mathrm{DMT}$, TNS, BB, and BJH participated in the design of the study. DC, $\mathrm{KDR}, \mathrm{CH}, \mathrm{NS}, \mathrm{MSP}, \mathrm{DMT}$ and TNS contributed to data collection and organization. BJH performed the statistical analysis. TNS, KDR, DMT, and $\mathrm{BB}$ conceived of the study, and participated in its design and coordination. The manuscript was drafted with the help of multiple authors: KDR, CH, BJH, NS, DC, $\mathrm{BL}$, and TNS. All authors read and approved the final manuscript.

Ethics approval and consent to participate

The institutional health sciences research board at the University of Virginia waived the need for approval and consent for this study.

Consent for publication

Not applicable.

\section{Competing interests}

The authors declare that they have no competing interests.

\section{Publisher's Note}

Springer Nature remains neutral with regard to jurisdictional claims in published maps and institutional affiliations.

\section{Author details}

${ }^{1}$ Department of Radiation Oncology, University of Virginia School of Medicine, 1240 Lee Street, Box 800383, Charlottesville, VA 22908, USA. ${ }^{2}$ Division of Translational Research and Applied Statistics, Department of Public Health Sciences, University of Virginia School of Medicine,

Charlottesville, VA, USA.

Received: 17 April 2018 Accepted: 10 July 2018

Published online: 16 July 2018

References

1. Siegel RL, Miller KD, Jemal A. Cancer statistics, 2017. CA Cancer J Clin. 2017;67(1):7-30.

2. National Comprehensive Cancer Network, NCCN Clinical Practice Guidelines in Oncology: Cervical Cancer v1.2016. [cited 2016 September 19]; Available from: http://www.nccn.org/professionals/physician_gls/pdf/cervical.pdf.

3. Tanderup K, Eifel PJ, Yashar CM, et al. Curative radiation therapy for locally advanced cervical cancer: brachytherapy is NOT optional. Int J Radiat Oncol Biol Phys. 2014;88(3):537-9.

4. Han K, Milosevic M, Fyles A, et al. Trends in the utilization of brachytherapy in cervical cancer in the United States. Int J Radiat Oncol Biol Phys. 2013; 87(1):111-9.

5. Patankar SS, Tergas Al, Deutsch I, et al. High versus low-dose rate brachytherapy for cervical cancer. Gynecol Oncol. 2015;136(3):534-41.

6. Romano KD, Pugh KJ, Trifiletti DM, et al. Transition from LDR to HDR brachytherapy for cervical cancer: evaluation of tumor control, survival, and toxicity. Brachytherapy. 2017;16(2):378-86.

7. Viswanathan AN, Beriwal S, De Los Santos JF, et al. American brachytherapy society consensus guidelines for locally advanced carcinoma of the cervix. Part II: high-dose-rate brachytherapy. Brachytherapy. 2012;11(1):47-52.

8. Viswanathan AN, Thomadsen BC, et al. American Brachytherapy Society cervical Cancer recommendations. American Brachytherapy Society consensus guidelines for locally advanced carcinoma of the cervix. Part I: general principles. Brachytherapy. 2012;11(1):33-46.

9. Haie-Meder C, Potter R, Van Limbergen E, et al. Recommendations from Gynaecological (GYN) GEC-ESTRO working group (I): concepts and terms in 
3D image based 3D treatment planning in cervix cancer brachytherapy with emphasis on MRI assessment of GTV and CTV. Radiother Oncol. 2005; 74(3):235-45.

10. Potter R, Tanderup K, Kirisits C, et al. The EMBRACE II study: the outcome and prospect of two decades of evolution within the GEC-ESTRO GYN working group and the EMBRACE studies. Clin Transl Radiat Oncol. 2018;9:48-60.

11. Sturdza A, Potter R, Fokdal LU, et al. Image guided brachytherapy in locally advanced cervical cancer: improved pelvic control and survival in RetroEMBRACE, a multicenter cohort study. Radiother Oncol. 2016;120(3):428-33.

12. National Insitutes of Health, N.C.I. Common Terminology Criteria for Adverse Events(CTCAE) version 4.0. [cited 2015 July 22]; Available from: https://evs. nci.nih.gov/ftp1/CTCAE/CTCAE_4.03/Archive/CTCAE_4.0_2009-05-29_ QuickReference $8.5 \times 11 . p d f$

13. Trifiletti DM, Libby B, Feuerlein S, et al. Implementing MRI-based target delineation for cervical cancer treatment within a rapid workflow environment for image-guided brachytherapy: a practical approach for centers without in-room MRI. Brachytherapy. 2015;14(6):905-9.

14. Mazeron R, Fokdal LU, Kirchheiner K, et al. Dose-volume effect relationships for late rectal morbidity in patients treated with chemoradiation and MRIguided adaptive brachytherapy for locally advanced cervical cancer: results from the prospective multicenter EMBRACE study. Radiother Oncol. 2016; 120(3):412-9.

15. Georg P, Potter R, Georg D, et al. Dose effect relationship for late side effects of the rectum and urinary bladder in magnetic resonance imageguided adaptive cervix cancer brachytherapy. Int I Radiat Oncol Biol Phys. 2012;82(2):653-7.

16. Mayadev J, Viswanathan A, Liu Y, et al. American brachytherapy task group report: a pooled analysis of clinical outcomes for high-dose-rate brachytherapy for cervical cancer. Brachytherapy. 2017;16(1):22-43.

17. Ribeiro I, Janssen $H$, De Brabandere $M$, et al. Long term experience with $3 D$ image guided brachytherapy and clinical outcome in cervical cancer patients. Radiother Oncol. 2016;120(3):447-54.

18. Tharavichtikul E, Meungwong P, Chitapanarux T, et al. The association of rectal equivalent dose in 2 Gy fractions (EQD2) to late rectal toxicity in locally advanced cervical cancer patients who were evaluated by rectosigmoidoscopy in Faculty of Medicine Chiang Mai University. Radiat Oncol J. 2014;32(2):57-62.

19. Tanderup, K., R. Potter, J.C. Lindegaard, et al. EMBRACE II study protocol v.1. 0. 2018 [cited 2018; Available from: https://www.embracestudy.dk/ UserUpload/PublicDocuments/EMBRACE\%20||\%20Protocol.pdf.

20. Kim Y, Kim YJ, Kim JY, et al. Toxicities and dose-volume histogram parameters of MRI-based brachytherapy for cervical cancer. Brachytherapy. 2017;16(1):116-25.

21. Zakariaee R, Hamarneh G, Brown CJ, et al. Bladder accumulated dose in imageguided high-dose-rate brachytherapy for locally advanced cervical cancer and its relation to urinary toxicity. Phys Med Biol. 2016;61 (24):8408-24.

22. Kirchheiner K, Nout RA, Lindegaard JC, et al. Dose-effect relationship and risk factors for vaginal stenosis after definitive radio(chemo)therapy with image-guided brachytherapy for locally advanced cervical cancer in the EMBRACE study. Radiother Oncol. 2016;118(1):160-6.

23. Charra-Brunaud C, Levitchi M, Delannes M, et al., Dosimetric and clinical results of a French prospective study of $3 \mathrm{D}$ brachytherapy for cervix carcinoma. Radiother Oncol. 2011;99:S57.

24. Potter R, Georg P, Dimopoulos JC, et al. Clinical outcome of protocol based image (MRl) guided adaptive brachytherapy combined with 3D conformal radiotherapy with or without chemotherapy in patients with locally advanced cervical cancer. Radiother Oncol. 2011;100(1):116-23.

25. Viswanathan AN, Erickson B, Gaffney DK, et al. Comparison and consensus guidelines for delineation of clinical target volume for CT- and MR-based brachytherapy in locally advanced cervical cancer. Int J Radiat Oncol Biol Phys. 2014;90(2):320-8.

26. Harmon G, Chinsky B, Surucu M, et al. Bladder distension improves the dosimetry of organs at risk during intracavitary cervical high-dose-rate brachytherapy. Brachytherapy. 2016;15(1):30-4

27. Gaudet M, Lim P, Yuen C, et al. Comparative analysis of rectal dose parameters in image-guided high-dose-rate brachytherapy for cervical cancer with and without a rectal retractor. Brachytherapy. 2014;13(3):257-62.

Ready to submit your research? Choose BMC and benefit from:

- fast, convenient online submission

- thorough peer review by experienced researchers in your field

- rapid publication on acceptance

- support for research data, including large and complex data types

- gold Open Access which fosters wider collaboration and increased citations

- maximum visibility for your research: over $100 \mathrm{M}$ website views per year

At BMC, research is always in progress.

Learn more biomedcentral.com/submissions 